\title{
Analysis of a Viral Infection Model with Delayed Nonlytic Immune Response
}

\author{
Mengye Chen, ${ }^{1}$ Liang You, ${ }^{1}$ Jie Tang, ${ }^{1}$ Shasha Su, ${ }^{2}$ and Ruiming $\mathrm{Zhang}^{1}$ \\ ${ }^{1}$ College of Science, Northwest A\&F University, Yangling, Shaanxi 712100, China \\ ${ }^{2}$ School of Economics and Management, Xidian University, Xian, Shaanxi 710071, China \\ Correspondence should be addressed to Ruiming Zhang; ruimingzhang@yahoo.com
}

Received 6 June 2014; Accepted 26 September 2014

Academic Editor: Kaifa Wang

Copyright (c) 2015 Mengye Chen et al. This is an open access article distributed under the Creative Commons Attribution License, which permits unrestricted use, distribution, and reproduction in any medium, provided the original work is properly cited.

\begin{abstract}
We investigate the dynamical behavior of a virus infection model with delayed nonlytic immune response. By analyzing corresponding characteristic equations, the local stabilities of two boundary equilibria are established. By using suitable Lyapunov functional and LaSalle's invariance principle, we establish the global stability of the infection-free equilibrium. We find that the infection free equilibrium $E_{0}$ is globally asymptotically stable when $R_{0} \leqslant 1$, and the infected equilibrium without immunity $E_{1}$ is local asymptotically stable when $1<R_{0} \leqslant 1+b \beta / c d$. Under the condition $R_{0}>1+b \beta / c d$ we obtain the sufficient conditions to the local stability of the infected equilibrium with immunity $E_{2}$. We show that the time delay can change the stability of $E_{2}$ and lead to the existence of Hopf bifurcations. The stabilities of bifurcating periodic solutions are studied and numerical simulations to our theorems are provided.
\end{abstract}

\section{Introduction}

Mathematical models have been proven valuable in understanding the population dynamics of viral load in vivo. A proper model may play significant role in a better understanding of the disease and the various drug therapy strategies. Viral infection models have received great attention in recent years [1-7]. In most viral infections, cytotoxic $\mathrm{T}$ lymphocytes (CTLs) play a critical role in antiviral defense by providing a cell-mediated response to specific foreign antigens associated with cells. Therefore, the immune response after a viral infection is universal and necessary to eliminate or control the disease.

Recently, there have been a lot of papers on virus dynamics within host; some include the immune response directly [8-13]. During viral infections, the host immune system reacts with innate and antigen-specific immune response. Both types of response can be subdivided broadly into lytic and nonlytic components. Lytic components kill infected cells, whereas the nonlytic components inhibit viral replication through soluble mediators. As a part of the innate response, cytotoxic T lymphocytes (CTLs) kill infected cells, while antibodies neutralize free virus particles and thus inhibit the infection of susceptible cells. In addition, CD4+ and CD8+ T cells can secrete cytokines that inhibit viral replication [12]. In order to investigate the role of direct lytic and nonlytic inhibition of viral replication by immune cells in viral infections, a mathematical model was constructed to describe the basic dynamics of the interaction among susceptible host cells, a virus population, and immune response, which is described by the following differential equations $[14,15]$ :

$$
\begin{gathered}
x^{\prime}(t)=s-d x-\frac{\beta x y}{1+q z}, \\
y^{\prime}(t)=\frac{\beta x y}{1+q z}-a y-p y z, \\
z^{\prime}(t)=c y-b z,
\end{gathered}
$$

where $x(t), y(t)$, and $z(t)$ represent the densities of uninfected target cells, virus, and CTL cells at time $t$, respectively. Uninfected cells are produced at rate $s$, die at rate $d x$, and become infected by virus at rate $\beta x y$ without the immune 
response; to model nonlytic antiviral, viral replication is inhibited by the immune response at rate $1+q z$; infected cells die at rate $a y$ and are removed at rate $p y z$ by the CTL immune response. The virus-specific CTL cells proliferate at rate $c y$ by contact with infected cells and die at rate $b z$. The parameter $p$ expresses the strength of the lytic component; the parameter $q$ expresses the efficacy of the nonlytic component.

Time delays cannot be ignored in models for immune response. Antigenic stimulation generating CTLs may need a period of time $\tau$ [16-18]; the CTL response at time $t$ may depend on the population of antigen at a previous time $t-\tau$. Under the assumption of retarded immune response, Wang et al. [18] studied the effects of the time delay for immune response and assumed the time evolution of the population of CTL cells $z$ is governed by the delayed nonlinear differential equation $z^{\prime}(t)=c y(t-\tau)-b z$. Li and Shu [19] and Xie et al. [20] investigated the effects of a time delay on a threedimensional system with $z^{\prime}(t)=c y(t-\tau) z(t-\tau)$. However, both studies do not consider the nonlytic component. In this paper we propose a more general model,

$$
\begin{gathered}
x^{\prime}(t)=s-d x-\frac{\beta x y}{1+q z}, \\
y^{\prime}(t)=\frac{\beta x y}{1+q z}-a y-p y z, \\
z^{\prime}(t)=c y(t-\tau) z(t-\tau)-b z,
\end{gathered}
$$

with the initial conditions

$$
\left(x(0), y_{0}(\theta), z_{0}(\theta)\right) \in \mathbb{R}_{+} \times \mathbb{C}^{+} \times \mathbb{C}^{+},
$$

where $\mathbb{C}^{+}=\mathbb{C}\left([-\tau, 0], \mathbb{R}_{+}\right)$which is the Banach space of continuous functions mapping the interval $[-\tau, 0]$ into $\mathbb{R}_{+}$with the topology of uniform convergence. Clearly, the models studied in [18-20] correspond to the case $q=0$ in our general model (2). In this work we study (2) in the more general case $q \geqslant 0$.

This paper is organized as follows. In Section 2, we study the local asymptotic stability of the infection-free equilibrium and the immune-exhausted equilibrium of system (2), and the global asymptotic stability of the infection-free equilibrium also is investigated. In Section 3, we analyze the local stability of the positive equilibrium and the existence of Hopf bifurcations. In Section 4, the direction and stability of Hopf bifurcation are analyzed by the normal form theory and center manifold approach. In Section 5, we present numerical simulations to illustrate our results. Finally, in Section 6 we provide concluding remarks.

\section{The Stability Analysis of Equilibrium}

Considering the existence of the three equilibria, then we have the following conclusions.

Proposition 1. Let $R_{0}=s \beta / a d$. Then, consider the following.

(1) If $R_{0}<1$, system (2) only has an infection-free equilibrium $E_{0}=\left(x_{0}, 0,0\right)$, where $x_{0}=s / d$.
(2) If $1<R_{0} \leqslant 1+b \beta / c d$, in addition to the infectionfree equilibrium $E_{0}$, system (2) has another unique equilibrium $E_{1}\left(x_{1}, y_{1}, 0\right)$ where $x_{1}=a / \beta$ and $y_{1}=$ $(s \beta-a d) / a \beta$.

(3) If $R_{0}>1+b \beta / c d$, in addition to the infectionfree equilibrium $E_{0}$, system (2) has another infected equilibrium $E_{2}=\left(x_{2}, y_{2}, z_{2}\right)$ that corresponds to the survival of free virus and CTL, where

$$
\begin{gathered}
x_{2}=\frac{-1}{d c}\left(b p z_{2}+a b-s c\right), \\
y_{2}=\frac{b}{c},
\end{gathered}
$$

$z_{2}$

$$
\begin{aligned}
& =\left(\sqrt{(a c d q+b \beta p+c d p)^{2}-4 c d p q(a b \beta+a c d-\beta c s)}\right. \\
& \quad-a c d q-b \beta p-c d p) \\
& \times(2 c d p q)^{-1} .
\end{aligned}
$$

By the similar method in [20], we have the following result.

Theorem 2. Under the above initial conditions (3), all solutions of system (2) are positive for $t>0$ and there exists $M>0$, such that all the solutions satisfy $x(t)<M, y(t)<M$, and $z(t)<M$ for all large $t$.

Theorem 3. If $R_{0}<1$, then the infection-free equilibrium $E_{0}$ is locally asymptotically stable, and $E_{0}$ is unstable if $R_{0}>1$.

Proof. The characteristic equation about $E_{0}$ is given by

$$
\left|\begin{array}{ccc}
d+\lambda & \frac{s \beta}{d} & 0 \\
0 & a+\lambda-\frac{s \beta}{d} & 0 \\
0 & 0 & b+\lambda
\end{array}\right|=0
$$

that is,

$$
(\lambda+b)(\lambda+d)\left(\lambda+a-\frac{\beta s}{d}\right)=0 .
$$

It is clear that (6) has negative roots $\lambda_{1}=-d$ and $\lambda_{2}=-b$. If $R_{0}<1$, then $\lambda_{3}=(s \beta-a d) / d<(a d-a d) / d=0$. This shows that all roots of (6) have the negative real part; infection-free equilibrium $E_{0}$ is locally asymptotically stable. If $R_{0}>1$, then $\lambda_{3}>0$. Equation (6) has a positive root; infection-free equilibrium $E_{0}$ is unstable.

Theorem 4. If $1<R_{0} \leqslant 1+b \beta / c d$, then immune-exhausted equilibrium $E_{1}\left(x_{1}, y_{1}, 0\right)$ is locally asymptotically stable, and $E_{1}$ is unstable if $R_{0}>1+b \beta / c d$. 
Proof. The characteristic equation about $E_{1}$ is given by

$$
\left|\begin{array}{ccc}
d+y_{1} \beta+\lambda & a & -a q y_{1} \\
-y_{1} \beta & \lambda & p y_{1}+a q y_{1} \\
0 & 0 & b-c e^{-\lambda \tau} y_{1}+\lambda
\end{array}\right|=0
$$

that is,

$$
\left(a \beta y_{1}+d \lambda+\lambda^{2}+\beta \lambda y_{1}\right)\left(b-c y_{1} e^{-\lambda \tau}+\lambda\right)=0 .
$$

It is easy to see that

$$
\begin{aligned}
& \lambda_{1}+\lambda_{2}=-\frac{s \beta}{a}<0, \\
& \lambda_{1} \lambda_{2}=s \beta-a d>0 .
\end{aligned}
$$

If $R_{0}>1$, then $\lambda_{1}$ and $\lambda_{2}$ are both negative real roots, and $\lambda_{3}$ is determined by the following equation:

$$
f(\lambda) \triangleq\left(b-c y_{1} e^{-\lambda \tau}+\lambda\right)=0
$$

If $1<R_{0} \leqslant 1+b \beta / c d$ and $\tau=0$, then $\lambda_{3}=c y_{1}-b=$ $(-a b \beta-a c d+\beta c s) / a \beta<0$. Therefore, if $\tau=0$ and $1<R_{0} \leqslant$ $1+b \beta / c d$, then $E_{1}$ is locally asymptotically stable. If $\tau>0$, let $i \omega(\omega>0)$ be a solution of (10). Substituting $\lambda=i \omega$ into (10) and separating the real and imaginary parts, we have

$$
\begin{gathered}
b=c y_{1} \cos (\tau \omega) \\
\omega=-c y_{1} \sin (\tau \omega) .
\end{gathered}
$$

Squaring and adding the two equations of (11) together, we obtain $b^{2}+\omega^{2}=c^{2} y_{1}^{2}$, which has solution

$$
\omega^{2}=\frac{(c(a d-s \beta)-a b \beta)(a b \beta+a c d-\beta c s)}{a^{2} \beta^{2}} .
$$

Since $1<R_{0} \leqslant 1+b \beta / c d$, then we have $(a d-s \beta)<0$ and $(a b \beta+a c d-\beta c s) \geqslant 0$; thus we get $\omega^{2} \leqslant 0$, which contradicts with our original assumption $\omega>0$. Therefore, (10) must have no pure imaginary root. Hence, in the case of $1<R_{0} \leqslant 1+b \beta / c d, E_{1}$ is locally asymptotically stable for all $\tau \geqslant 0$. If $R_{0}>1+b \beta / c d$, then $f(0)=(a b \beta+a c d-\beta c s) / a \beta<0$, and $\lim _{\lambda \rightarrow+\infty} f(\lambda)=+\infty$. By the intermediate value theorem of continuous functions, we see that equation $f(\lambda)=0$ has at least one positive real root; thus the immune-exhausted equilibrium $E_{1}$ is unstable.

Theorem 5. If $R_{0} \leqslant 1$, the infection-free equilibrium $E_{0}\left(x_{0}, 0,0\right)$ is globally asymptotically stable.

Proof. Define the Lyapunov functional

$$
V_{1}(t)=x-x_{0}-x_{0} \ln \frac{x}{x_{0}}+y+\frac{p}{c} z+p \int_{t-\tau}^{t} y(\theta) z(\theta) \mathrm{d} \theta
$$

we calculate the derivative of $V_{1}(t)$ along positive solutions of system (2) then substitute $s$ for $s=d x_{0}$ to obtain

$$
\begin{aligned}
\frac{\mathrm{d} V_{1}(t)}{\mathrm{d} t}= & \left(1-\frac{x_{0}}{x(t)}\right)\left[-d\left(x(t)-x_{0}\right)-\frac{\beta x(t) y(t)}{q z(t)+1}\right] \\
& +\frac{\beta x(t) y(t)}{q z(t)+1}-a y(t)-\frac{p b}{c} z(t) \\
= & -d \frac{\left[x_{0}-x(t)\right]^{2}}{x(t)}-\frac{b p z(t)}{c} \\
& +\frac{y(t)[-a d q z(t)+\beta s-a d]}{d(q z(t)+1)} \\
= & -d \frac{\left[x_{0}-x(t)\right]^{2}}{x(t)}-\frac{b p z(t)}{c} \\
& +\frac{y(t)\left[-a d q z(t)+a d\left(R_{0}-1\right)\right]}{d(q z(t)+1)} .
\end{aligned}
$$

Clearly, in the case of $R_{0} \leqslant 1$, we have $\mathrm{d} V_{1}(t) / \mathrm{d} x \leq 0$ since $x(t), y(t)$, and $z(t)$ are positive, and $\mathrm{d} V_{1}(t) / \mathrm{d} x=0$ if and only if $(x, y, z)=\left(x_{0}, 0,0\right)$. Thus $E_{0}$ is globally asymptotically stable by Lyapunov-LaSalle invariance principle.

\section{The Stability of Infected Equilibrium and Hopf Bifurcation}

Let $y_{2} /\left(1+q z_{2}\right)=A$, apply $\beta x_{2} /\left(1+q z_{2}\right)=a+p z_{2}$ to the characteristic equation of system (2) at the positive equilibrium $E_{2}$, and we have

$$
\left|\begin{array}{ccc}
\lambda+d+A \beta & a+p z_{2} & -A q\left(a+p z_{2}\right) \\
-A \beta & \lambda & A\left(a q+p+2 p q z_{2}\right) \\
0 & -c e^{-\lambda \tau} z_{2} & \lambda-e^{-\lambda \tau} b+b
\end{array}\right|=0
$$

that is,

$$
P(\lambda, \tau)+Q(\lambda, \tau) e^{-\lambda \tau}=0
$$

where

$$
\begin{gathered}
P(\lambda, \tau)=\lambda^{3}+a_{1} \lambda^{2}+a_{2} \lambda+a_{3}, \\
Q(\lambda, \tau)=a_{4} \lambda^{2}+a_{5} \lambda+a_{6}, \\
a_{1}=A \beta+b+d, \\
a_{2}=a A \beta+A b \beta+A \beta p z_{2}+b d, \\
a_{3}=a A b \beta+A b \beta p z_{2}, \\
a_{5}=-b, \\
a_{6}=a A c d q z_{2}+A^{2} \beta c p q z_{2}^{2}+A^{2} \beta c p z_{2} \\
-A b \beta p z_{2}+2 A c d p q z_{2}^{2}+A c d p z_{2}-a A b \beta .
\end{gathered}
$$


If $\tau=0$, (16) becomes

$$
\lambda^{3}+\left(a_{1}+a_{4}\right) \lambda^{2}+\left(a_{2}+a_{5}\right) \lambda+a_{3}+a_{6}=0,
$$

where

$$
\begin{aligned}
a_{1}+a_{4}= & A \beta+d>0, a_{2}+a_{5} \\
= & a A \beta+a A c q z_{2}+2 A c p q z_{2}^{2} \\
& +A c p z_{2}+A \beta p z_{2}>0, a_{3}+a_{6} \\
= & a A c d q z_{2}+A^{2} \beta c p q z_{2}^{2}+A^{2} \beta c p z_{2} \\
& +2 A c d p q z_{2}^{2}+A c d p z_{2}>0 .
\end{aligned}
$$

Then,

$$
\begin{aligned}
\Delta_{1} & =a_{1}+a_{4}>0, \\
\Delta_{2} & =\left(a_{1}+a_{4}\right)\left(a_{2}+a_{5}\right)-\left(a_{3}+a_{6}\right) \\
& =A \beta\left(a+p z_{2}\right)\left(A \beta+A c q z_{2}+d\right)>0 .
\end{aligned}
$$

By the Routh-Hurwitz criterion, we know that all roots of (18) have negative real parts. From the above analysis, the following theorem holds.

Theorem 6. If $R_{0}>1+b \beta / c d$, the equilibrium $E_{2}$ is locally asymptotically stable for $\tau=0$.

From Theorem 6, when $\tau=0$, all roots of (16) lie to the left of imaginary axis. But, as $\tau$ is increased from zero, some of the roots may cross the imaginary axis to the right. Then the equilibrium $E_{2}$ becomes unstable. Now we suppose (16) has a pure imaginary root $\lambda=i \omega(\omega>0)$. Obviously, $\lambda=i \omega$ $(\omega>0)$ is a root of (16) if and only if $\omega$ satisfies

$$
\begin{aligned}
& -i \omega^{3}-a_{1} \omega^{2}+i a_{2} \omega+a_{3}+\left(-a_{4} \omega^{2}+i a_{5} \omega+a_{6}\right) \\
& \times(\cos (\tau \omega)-i \sin (\tau \omega))=0 .
\end{aligned}
$$

Separating the real and imaginary parts, we have

$$
\begin{aligned}
& a_{3}-a_{1} \omega^{2}=\left(a_{4} \omega^{2}-a_{6}\right) \cos (\tau \omega)-a_{5} \omega \sin (\tau \omega), \\
& \omega^{3}-a_{2} \omega=\left(a_{4} \omega^{2}-a_{6}\right) \sin (\tau \omega)+a_{5} \omega \cos (\tau \omega) .
\end{aligned}
$$

From (21), we also obtain

$$
\left|-i \omega^{3}-a_{1} \omega^{2}+i a_{2} \omega+a_{3}\right|^{2}=\left|-a_{4} \omega^{2}+i a_{5} \omega+a_{6}\right|^{2}\left|e^{-i \omega \tau}\right|^{2} .
$$

So we have

$$
\omega^{6}+b_{1} \omega^{4}+b_{2} \omega^{2}+b_{3}=0
$$

where

$$
\begin{gathered}
b_{1}=a_{1}^{2}-a_{4}^{2}-2 a_{2}, \\
b_{2}=a_{2}^{2}-a_{5}^{2}-2 a_{1} a_{3}+2 a_{4} a_{6}, \\
b_{3}=a_{3}^{2}-a_{6}^{2} .
\end{gathered}
$$

Denote

$$
G(x)=x^{3}+b_{1} x^{2}+b_{2} x+b_{3}
$$

Therefore, if (16) has a pure imaginary root $i \omega$, then equation

$$
G(x)=0
$$

has a positive real root $\omega^{2}$.

Suppose that (27) has $K, 1 \leqslant K \leqslant 3$, positive real roots, which are $x_{k}, 1 \leqslant k \leqslant K$, respectively. From (22), we have

$$
\begin{aligned}
& \cos \left(\tau \sqrt{x_{k}}\right)=Q_{k} \\
& =-\frac{a_{5} \sqrt{x_{k}}\left(x_{k}^{3 / 2}-a_{2} \sqrt{x_{k}}\right)-\left(a_{3}-a_{1} x_{k}\right)\left(a_{6}-a_{4} x_{k}\right)}{-\left(a_{6}-a_{4} x_{k}\right)^{2}-a_{5}^{2} x_{k}}, \\
& \sin \left(\tau \sqrt{x_{k}}\right)=P_{k} \\
& =\frac{\sqrt{x_{k}}\left(a_{4} x_{k}^{2}-a_{2} a_{4} x_{k}+a_{1} a_{5} x_{k}-a_{6} x_{k}-a_{3} a_{5}+a_{2} a_{6}\right)}{a_{5}^{2} x_{k}+a_{4}^{2} x_{k}^{2}-2 a_{4} a_{6} x_{k}+a_{6}^{2}} .
\end{aligned}
$$

Let

$$
\tau_{k}^{(j)}= \begin{cases}\frac{1}{\sqrt{x_{k}}}\left[\arccos \left(Q_{k}\right)+2 j \pi\right], & \text { if } P_{n} \geqslant 0, \\ \frac{1}{\sqrt{x_{k}}}\left[2 \pi-\arccos \left(Q_{k}\right)+2 j \pi\right], & \text { if } P_{n}<0,\end{cases}
$$

where $1 \leqslant k \leqslant K$ and $j=0,1,2, \ldots$. Then $\pm i \sqrt{x_{k}}$ is a pair of pure imaginary roots of (16).

Theorem 7 (see [21]). Suppose the characteristic equation is the form

$$
f_{0}(s)+f_{1}(s) e^{-s \tau}=0,
$$

where $f_{0}$ and $f_{1}$ are continuously differentiable with respect to $s$. One of the roots to $(31)$ is $s(\tau)=\alpha(\tau)+i \omega(\tau)$, where $s(\tau)$ is continuously differentiable with respect to $\tau$, satisfying $\alpha\left(\tau_{0}\right)=$ 0 and $\omega\left(\tau_{0}\right)$ for a positive real number $\tau_{0}$. Denote $\Phi(\omega)=$ $\left|f_{0}(i \omega)\right|^{2}-\left|f_{1}(i \omega)\right|^{2}$; then we have $\operatorname{sign}\left[\operatorname{dRe}(s) /\left.\mathrm{d} \tau\right|_{\tau=\tau_{0}}\right]=$ $\operatorname{sign}\left[\left.((1 / 2 \omega) \cdot(\mathrm{d} \Phi / \mathrm{d} \omega))\right|_{\omega=\omega_{0}}\right]$.

Let $\lambda(\tau)=\xi(\tau)+i \omega(\tau)$ be the root of (16) near $\tau=\tau_{k}^{(j)}$ satisfying $\xi\left(\tau_{k}^{(j)}\right)=0, \omega\left(\tau_{k}^{(j)}\right)=\sqrt{x_{k}}$. Then, by Theorem 7 , we have the following theorem.

Theorem 8. $\mathrm{d} \xi /\left.\mathrm{d} \tau\right|_{\tau=\tau_{k}^{(j)}}$ and $\mathrm{d} G /\left.\mathrm{d} x\right|_{x=x_{k}}$ have the same sign.

We choose $\tau_{0}=\min \left\{\tau_{k}^{(j)} \mid 1 \leqslant k \leqslant K, j=0,1,2, \ldots\right\}$, where $\tau_{k}^{(j)}$ is defined by (30). Summarizing the above analysis and combining [22], the following theorem holds.

Theorem 9. (1) For system (2), its equilibrium $E_{2}$ is asymptotically stable for $\tau \in\left[0, \tau_{0}\right)$ if (27) has some positive real roots;

(2) if $x_{k_{0}}$ is a simple positive real root of (27), then system (2) undergoes a Hopf bifurcation at the equilibrium $E_{2}$ when $\tau=\tau_{0}$. 


\section{Direction and Stability of Hopf Bifurcation}

In this section, we will study the direction of the Hopf bifurcations and stability of bifurcating periodic solutions by applying the normal theory and the center manifold theorem introduced in [22]. We always assume that system (2) undergoes Hopf bifurcation at the positive equilibrium $E_{2}$ for $\tau=\tau_{0}$, and then $\pm i \omega$ are pure imaginary roots of the characteristic equation at the positive equilibrium $E_{2}$.

Let $u_{1}(t)=x(\tau t)-x^{*}, u_{2}(t)=y(\tau t)-y^{*}$, and $u_{3}(t)=$ $z(\tau t)-z^{*}, \tau=\tau_{0}+v$; then $\nu=0$ is the Hopf bifurcation value. The Taylor expansion of (2) at the equilibrium point is given by

$$
\begin{aligned}
& \frac{\mathrm{d} u_{1}(t)}{\mathrm{d} t} \\
& =\left(\tau_{0}+\nu\right) \\
& \times\left\{-\left(d+\frac{\beta y^{*}}{q z^{*}+1}\right) u_{1}-\frac{\beta x^{*}}{q z^{*}+1} u_{2}+\frac{\beta q x^{*} y^{*}}{\left(q z^{*}+1\right)^{2}} u_{3}\right. \\
& \left.+\frac{\beta q u_{1} u_{3} y^{*}}{\left(q z^{*}+1\right)^{2}}-\frac{\beta u_{1} u_{2}}{q z^{*}+1}+\frac{\beta q x^{*} u_{2} u_{3}}{\left(1+q z^{*}\right)^{2}}+\cdots\right\}, \\
& \frac{\mathrm{d} u_{2}(t)}{\mathrm{d} t} \\
& =\left(\tau_{0}+v\right) \\
& \times\left\{\frac{\beta y^{*}}{q z^{*}+1} u_{1}-\left(p y^{*}+\frac{\beta q x^{*} y^{*}}{\left(q z^{*}+1\right)^{2}}\right) u_{3}\right. \\
& -\left(p+\frac{\beta q x^{*}}{\left(q z^{*}+1\right)^{2}}\right) u_{2} u_{3} \\
& \left.-\frac{\beta q y^{*} u_{1} u_{3}}{\left(q z^{*}+1\right)^{2}}+\frac{\beta u_{1} u_{2}}{q z^{*}+1}+\cdots\right\} \text {, } \\
& \frac{\mathrm{d} u_{3}(t)}{\mathrm{d} t}=\left(\tau_{0}+\nu\right)\left\{-b u_{3}+c z^{*} u_{2}(t-1)+c y^{*} u_{3}(t-1)\right. \\
& \left.+c u_{3}(t-1) u_{2}(t-1)\right\} .
\end{aligned}
$$

We denote the above system by

$$
\dot{u}(t)=L_{\nu}\left(u_{t}\right)+f\left(v, u_{t}\right),
$$

where $u(t)=\left(u_{1}(t), u_{2}(t), u_{3}(t)\right)^{\top} \in \mathbb{R}^{3}$, and the maps $L_{v}$ : $\mathbb{C} \rightarrow \mathbb{R}^{3}, f: \mathbb{R} \times \mathbb{C} \rightarrow \mathbb{R}^{3}$ are given by

$$
\begin{aligned}
& L_{\nu}(\phi) \\
& =\left(\tau_{0}+\nu\right) \\
& \quad \times\left(\begin{array}{ccc}
-\left(d+\frac{\beta y^{*}}{q z^{*}+1}\right) & -\frac{\beta x^{*}}{q z^{*}+1} & \frac{\beta q x^{*} y^{*}}{\left(q z^{*}+1\right)^{2}} \\
\frac{\beta y^{*}}{q z^{*}+1} & 0 & -\left(p y^{*}+\frac{\beta q x^{*} y^{*}}{\left(q z^{*}+1\right)^{2}}\right) \\
0 & 0 & -b
\end{array}\right)
\end{aligned}
$$

$$
\begin{aligned}
& \times\left(\begin{array}{l}
\phi_{1}(0) \\
\phi_{2}(0) \\
\phi_{3}(0)
\end{array}\right) \\
& +\left(\tau_{0}+v\right)\left(\begin{array}{ccc}
0 & 0 & 0 \\
0 & 0 & 0 \\
0 & c z^{*} & c y^{*}
\end{array}\right)\left(\begin{array}{l}
\phi_{1}(-1) \\
\phi_{2}(-1) \\
\phi_{3}(-1)
\end{array}\right), \\
& f(\nu, \phi) \\
& =\left(\tau_{0}+\nu\right)\left\{\left(\begin{array}{c}
-\frac{\beta \phi_{1}(0) \phi_{2}(0)}{q z^{*}+1} \\
\frac{\beta \phi_{1}(0) \phi_{2}(0)}{q z^{*}+1} \\
0
\end{array}\right)\right. \\
& +\left(\begin{array}{c}
\frac{\beta q y^{*} \phi_{1}(0) \phi_{3}(0)}{\left(q z^{*}+1\right)^{2}} \\
\frac{-\beta q y^{*} \phi_{1}(0) \phi_{3}(0)}{\left(q z^{*}+1\right)^{2}} \\
0
\end{array}\right) \\
& \left.+\left(\begin{array}{c}
\frac{\beta q x^{*} \phi_{2}(0) \phi_{3}(0)}{\left(q z^{*}+1\right)^{2}} \\
\frac{-\left(\beta q x^{*}+p\right) \phi_{2}(0) \phi_{3}(0)}{\left(q z^{*}+1\right)^{2}} \\
c \phi_{2}(-1) \phi_{3}(-1)
\end{array}\right)+\cdots\right\} .
\end{aligned}
$$

By the Riesz representation theorem, there exists a function $\eta(\theta, v)$ of bounded variation for $\theta \in[0,1]$ such that

$$
L_{\nu}(\phi)=\int_{-1}^{0} \phi(\theta) \mathrm{d} \eta(\theta, \nu), \quad \phi \in C .
$$

In fact, we can choose

$$
\begin{aligned}
& \eta(\theta, \nu) \\
& =\left(\tau_{0}+\nu\right) \\
& \times\left(\begin{array}{ccc}
-\left(d+\frac{\beta y^{*}}{q z^{*}+1}\right) & -\frac{\beta x^{*}}{q z^{*}+1} & \frac{\beta q x^{*} y^{*}}{\left(q z^{*}+1\right)^{2}} \\
\frac{\beta y^{*}}{q z^{*}+1} & 0 & -\left(p y^{*}+\frac{\beta q x^{*} y^{*}}{\left(q z^{*}+1\right)^{2}}\right) \\
0 & 0 & -b
\end{array}\right) \\
& \times \delta(\theta)-\left(\tau_{0}+\nu\right)\left(\begin{array}{ccc}
0 & 0 & 0 \\
0 & 0 & 0 \\
0 & c z^{*} & c y^{*}
\end{array}\right) \delta(\theta+1),
\end{aligned}
$$

where $\delta$ denote the Dirac delta function. For $\phi \in \mathbb{C}([-1,0]$, $\left.\mathbb{R}^{3}\right)$, let 


$$
\begin{gathered}
A(\nu) \phi(\theta)= \begin{cases}\frac{\mathrm{d} \phi(\theta)}{\mathrm{d} \theta}, & \text { if } \theta \in[-1,0), \\
\int_{-1}^{0} \mathrm{~d} \eta(\xi, \nu) \phi(\xi), & \text { if } \theta=0 ;\end{cases} \\
R(\nu) \phi(\theta)= \begin{cases}0, & \text { if } \theta \in[-1,0), \\
f(\nu, \phi), & \text { if } \theta=0,\end{cases}
\end{gathered}
$$

then system (33) is equivalent to

$$
\dot{u}(t)=A(\nu) u_{t}(\theta)+R(\nu) u_{t}(\theta)
$$

where $u_{t}(\theta)=u(t+\theta)$ for $\theta \in[-1,0]$.

For $\varphi \in \mathbb{C}\left([-1,0], \mathbb{R}^{3}\right)$, the adjoint operator of $A(0)$ is defined by

$$
A^{*}(0) \varphi(s)= \begin{cases}-\frac{\mathrm{d} \varphi(s)}{\mathrm{d} s}, & \text { if } s \in(0,1], \\ \int_{-1}^{0} \mathrm{~d} \eta^{\top}(\xi, 0) \varphi(-\xi), & \text { if } s=0,\end{cases}
$$

and define a bilinear inner product

$$
\begin{aligned}
& \langle\varphi(s), \phi(\theta)\rangle \\
& \quad=\bar{\varphi}^{\top}(0) \phi(0)-\int_{-1}^{0} \int_{\xi=0}^{\theta} \bar{\varphi}^{\top}(\xi-\theta) \mathrm{d} \eta(\theta) \phi(\xi) \mathrm{d} \xi
\end{aligned}
$$

where $\eta(\theta)=\eta(\theta, 0)$. By the discussion in Section 3 we know that $\pm i \omega \tau_{0}$ are eigenvalues of $A(0)$. Hence, they are also eigenvalues of $A^{*}$. We define $q(\theta)$ and $q^{*}(s)$ to be the eigenvectors of $A$ and $A^{*}$ corresponding to the eigenvalues $i \omega \tau_{0}$ and $-i \omega \tau_{0}$, respectively.

Assume that $q(\theta)=\left(1, q_{1}, q_{2}\right)^{\top} e^{i \omega \tau_{0} \theta}$ is the eigenvector of $A(0)$ corresponding to $i \omega \tau_{0}$; then $A(0) q(\theta)=i \omega \tau_{0} q(\theta)$. It follows from the definition of $A(0)$ that we have

$$
\begin{aligned}
& \tau_{0}\left(\begin{array}{ccc}
i \omega+\left(d+\frac{\beta y^{*}}{q z^{*}+1}\right) & \frac{\beta x^{*}}{q z^{*}+1} & \frac{-\beta q x^{*} y^{*}}{\left(q z^{*}+1\right)^{2}} \\
-\frac{\beta y^{*}}{q z^{*}+1} & i \omega & \left(p y^{*}+\frac{\beta q x^{*} y^{*}}{\left(q z^{*}+1\right)^{2}}\right) \\
0 & -c z^{*} e^{-i \omega \tau_{0}} & i \omega+b-c y^{*} e^{-i \omega \tau_{0}}
\end{array}\right) q(0) \\
& =\left(\begin{array}{l}
0 \\
0 \\
0
\end{array}\right) .
\end{aligned}
$$

Then we obtain

$q_{1}$

$$
\begin{gathered}
=-\left(( q z ^ { * } + 1 ) \left(p\left(q z^{*}+1\right)\left(\beta y^{*}+(d+i \omega)\left(q z^{*}+1\right)\right)\right.\right. \\
\left.+\beta q x^{*}(d+i \omega)\right) \\
\times\left(\beta x^{*}\left(\beta q x^{*}+\left(q z^{*}+1\right)\left(p q z^{*}+p+i q \omega\right)\right)\right)^{-1},
\end{gathered}
$$

$q_{2}$

$$
\begin{aligned}
& =\left(i ( q z ^ { * } + 1 ) \left(\omega(d+i \omega)\left(q z^{*}+1\right)^{2}\right.\right. \\
& \left.\left.+\beta y^{*}\left(q \omega z^{*}-i \beta x^{*}+\omega\right)\right)\right) \\
& \times\left(\beta x^{*} y^{*}\left(\beta q x^{*}+\left(q z^{*}+1\right)\left(p q z^{*}+p+i q \omega\right)\right)\right)^{-1} .
\end{aligned}
$$

Similarly, let $q^{*}(s)=D\left(1, q_{1}^{*}, q_{2}^{*}\right)^{\top} e^{i \omega \tau_{0} s}$ be the eigenvector of $A^{*}$ corresponding to $-i \omega \tau_{0}$; by the definition of $A^{*}$ we get

$$
\begin{gathered}
q_{1}^{*}=\frac{\beta y^{*}+(d-i \omega)\left(q z^{*}+1\right)}{\beta y^{*}}, \\
q_{2}^{*}=-\left(i e ^ { - i \tau _ { 0 } \omega } \left(\omega(d-i \omega)\left(q z^{*}+1\right)^{2}+\beta y^{*}\right.\right. \\
\left.\times\left(q \omega z^{*}+i \beta x^{*}+\omega\right)\right) \\
\left.\times\left(\beta c y^{*} z^{*}\left(q z^{*}+1\right)\right)^{-1}\right) .
\end{gathered}
$$

In order to assure $\left\langle q^{*}(s), q(\theta)\right\rangle=1$, we need to determine the value of $D$. From (40) we have

$$
\begin{aligned}
& \left\langle q^{*}(s), q(\theta)\right\rangle \\
& =\bar{D}\left(1, \overline{q_{1}^{*}}, \overline{q_{2}^{*}}\right)\left(1, q_{1}, q_{2}\right)^{\top} \\
& -\int_{-1}^{0} \int_{\xi=0}^{\theta} \bar{D}\left(1, \overline{q_{1}^{*}}, \overline{q_{2}^{*}}\right) e^{-i \omega \tau_{0}(\xi-\theta)} \mathrm{d} \eta(\theta) \\
& \times\left(1, q_{1}, q_{2}\right)^{\top} e^{i \omega \tau_{0} \xi} \mathrm{d} \xi \\
& =\bar{D}\left\{1+q_{1} \overline{q_{1}^{*}}+q_{2} \overline{q_{2}^{*}}-\int_{-1}^{0}\left(1, \overline{q_{1}^{*}}, \overline{q_{2}^{*}}\right) \theta e^{i \omega \tau_{0} \theta}\right. \\
& \left.\times[\mathrm{d} \eta(\theta)]\left(1, q_{1}, q_{2}\right)^{\top}\right\} \\
& =\bar{D}\left\{\left(1+q_{1} \overline{q_{1}^{*}}+q_{2} \overline{q_{2}^{*}}\right)+\tau_{0} e^{-i \omega \tau_{0}}\left(1, \overline{q_{1}^{*}}, \overline{q_{2}^{*}}\right)\right. \\
& \left.\times\left(\begin{array}{ccc}
0 & 0 & 0 \\
0 & 0 & 0 \\
0 & c z^{*} & c y^{*}
\end{array}\right)\left(1, q_{1}, q_{2}\right)^{\top}\right\} \\
& =\bar{D}\left\{\left(1+q_{1} \overline{q_{1}^{*}}+q_{2} \overline{q_{2}^{*}}\right)+c \tau_{0} e^{-i \omega \tau_{0}} \overline{q_{2}^{*}}\right. \\
& \left.\times\left(z^{*} q_{1}+y^{*} q_{2}\right)\right\}=1 .
\end{aligned}
$$

Hence

$$
\bar{D}=\left[\left(1+q_{1} \overline{q_{1}^{*}}+q_{2} \overline{q_{2}^{*}}\right)+c \tau_{0} e^{-i \omega \tau_{0}} \overline{q_{2}^{*}}\left(z^{*} q_{1}+y^{*} q_{2}\right)\right]^{-1} .
$$


Using the method in [22] we obtain the following coefficients:

$$
\begin{aligned}
& g_{20}=2 \tau_{0} \bar{D}\left(F_{21} \overline{q_{1}^{*}}+F_{31} \overline{q_{2}^{*}}+F_{11}\right), \\
& g_{11}=\tau_{0} \bar{D}\left(F_{22} \overline{q_{1}^{*}}+F_{32} \overline{q_{2}^{*}}+F_{12}\right), \\
& g_{02}=2 \tau_{0} \bar{D}\left(F_{23} \overline{q_{1}^{*}}+F_{33} \overline{q_{2}^{*}}+F_{13}\right), \\
& g_{21}=2 \tau_{0} \bar{D}\left(F_{24} \overline{q_{1}^{*}}+F_{34} \overline{q_{2}^{*}}+F_{14}\right),
\end{aligned}
$$

where

$$
\begin{aligned}
& F_{11}=\beta\left(-q^{2}\right) q_{2}^{2} Q^{3} x^{*} y^{*}+Q^{2} \\
& \times\left(\beta q q_{1} q_{2} x^{*}+\beta q q_{2} y^{*}\right)-\beta q_{1} Q, \\
& F_{12}=-2 \beta q^{2} q_{2} Q^{3} x^{*} y^{*} \overline{q_{2}}+Q^{2} \\
& \times\left(\beta q q_{1} x^{*} \overline{q_{2}}+\beta q q_{2} x^{*} \overline{q_{1}}+\beta q y^{*} \overline{q_{2}}+\beta q q_{2} y^{*}\right) \\
& +Q\left(-\beta \overline{q_{1}}-\beta q_{1}\right) \text {, } \\
& F_{13}=\beta\left(-q^{2}\right) Q^{3} x^{*} y^{*}{\overline{q_{2}}}^{2}+Q^{2} \\
& \times\left(\beta q x^{*} \overline{q_{1} q_{2}}+\beta q y^{*} \overline{q_{2}}\right)-\beta Q \overline{q_{1}}, \\
& F_{14}=3 \beta q^{3} q_{2}^{2} Q^{4} x^{*} y^{*} \overline{q_{2}}+Q^{3} \\
& \times\left(-\beta q_{2}^{2} q^{2} x^{*} \overline{q_{1}}-2 \beta q_{1} q_{2} q^{2} x^{*} \overline{q_{2}}-2 \beta q_{2} q^{2} y^{*} \overline{q_{2}}\right. \\
& -\beta q_{2}^{2} q^{2} y^{*}-\beta q^{2} W_{320}(0) x^{*} y^{*} \overline{q_{2}} \\
& \left.-2 \beta q_{2} q^{2} W_{311}(0) x^{*} y^{*}\right) \\
& +Q^{2}\left(\beta q q_{1} \overline{q_{2}}+\beta q q_{2} \overline{q_{1}}+\beta q q_{2} q_{1}\right. \\
& +\frac{1}{2} \beta q W_{120}(0) y^{*} \overline{q_{2}} \\
& +\beta q q_{2} W_{111}(0) y^{*}+\frac{1}{2} \beta q W_{220}(0) x^{*} \overline{q_{2}} \\
& +\beta q q_{2} W_{211}(0) x^{*} \\
& +\beta q q_{1} W_{311}(0) x^{*}+\frac{1}{2} \beta q W_{320}(0) x^{*} \overline{q_{1}} \\
& \left.+\beta q W_{311}(0) y^{*}+\frac{1}{2} \beta q W_{320}(0) y^{*}\right) \\
& +Q\left(-\beta W_{211}(0)-\beta q_{1} W_{111}(0)\right. \\
& \left.-\frac{1}{2} \beta W_{220}(0)-\frac{1}{2} \beta W_{120}(0) \overline{q_{1}}\right), \\
& F_{21}=-p q_{1} q_{2}+\beta q^{2} q_{2}^{2} Q^{3} x^{*} y^{*}+Q^{2} \\
& \times\left(\beta(-q) q_{1} q_{2} x^{*}-\beta q q_{2} y^{*}\right)+\beta q_{1} Q \text {, }
\end{aligned}
$$

$$
\begin{aligned}
F_{22}= & -p q_{1} \overline{q_{2}}-p q_{2} \overline{q_{1}}+2 \beta q^{2} q_{2} Q^{3} x^{*} y^{*} \overline{q_{2}} \\
& +Q\left(\beta \overline{q_{1}}+\beta q_{1}\right) \\
& +Q^{2}\left(\beta(-q) q_{1} x^{*} \overline{q_{2}}-\beta q q_{2} x^{*} \overline{q_{1}}\right. \\
& \left.-\beta q y^{*} \overline{q_{2}}-\beta q q_{2} y^{*}\right), \\
F_{23}= & -p \overline{q_{1} q_{2}}+\beta q^{2} Q^{3} x^{*} y^{*}{\overline{q_{2}}}^{2}+Q^{2} \\
& \times\left(\beta(-q) x^{*} \overline{q_{1} q_{2}}-\beta q y^{*} \overline{q_{2}}\right)+\beta Q \overline{q_{1}},
\end{aligned}
$$

$F_{24}=-\frac{1}{2} p W_{220}(0) \overline{q_{2}}-3 \beta q^{3} q_{2}^{2} Q^{4} x^{*} y^{*} \overline{q_{2}}$

$$
-p q_{2} W_{211}(0)-\frac{1}{2} p W_{320}(0) \overline{q_{1}}-p q_{1} W_{311}(0)
$$$$
+Q\left(\frac{1}{2} \beta W_{120}(0) \overline{q_{1}}+\beta q_{1} W_{111}(0)\right.
$$$$
\left.+\beta W_{211}(0)+\frac{1}{2} \beta W_{220}(0)\right)
$$$$
+Q^{2}\left(-\beta q q_{2} q_{1}-\beta q q_{2} \overline{q_{1}}-\frac{1}{2} \beta q W_{120}(0) y^{*} \overline{q_{2}}\right.
$$$$
-\beta q q_{2} W_{111}(0) y^{*}-\beta q q_{2} W_{211}(0) x^{*}
$$$$
-\frac{1}{2} \beta q W_{220}(0) x^{*} \overline{q_{2}}-\frac{1}{2} \beta q W_{320}(0) x^{*} \overline{q_{1}}
$$$$
-\beta q q_{1} \overline{q_{2}}+\beta(-q) q_{1} W_{311}(0) x^{*}
$$$$
\left.-\beta q W_{311}(0) y^{*}-\frac{1}{2} \beta q W_{320}(0) y^{*}\right)
$$

$+Q^{3}\left(\beta q_{2}^{2} q^{2} x^{*} \overline{q_{1}}+2 \beta q_{1} q_{2} q^{2} x^{*} \overline{q_{2}}\right.$

$$
+2 \beta q_{2} q^{2} y^{*} \overline{q_{2}}+\beta q_{2}^{2} q^{2} y^{*}
$$$$
+2 \beta q^{2} q_{2} W_{311}(0) x^{*} y^{*}
$$$$
\left.+\beta q^{2} W_{320}(0) x^{*} y^{*} \overline{q_{2}}\right) \text {, }
$$

$$
F_{31}=c q_{1} q_{2} e^{-2 i \tau_{0} \omega},
$$$$
F_{32}=c q_{1} \overline{q_{2}}+c q_{2} \overline{q_{1}},
$$

$$
F_{33}=c \overline{q_{1} q_{2}} e^{2 i \tau_{0} \omega},
$$

$$
\begin{gathered}
F_{34}=\frac{1}{2} c W_{220}(-1) \overline{q_{2}} e^{i \tau_{0} \omega}+\frac{1}{2} c W_{320}(-1) \overline{q_{1}} e^{i \tau_{0} \omega} \\
+c q_{2} W_{211}(-1) e^{-i \tau_{0} \omega}+c q_{1} W_{311}(-1) e^{-i \tau_{0} \omega}, \\
Q=\frac{1}{1+q z^{*}},
\end{gathered}
$$




$$
\begin{gathered}
W_{120}=W_{20}^{(1)}, \quad W_{220}=W_{20}^{(2)}, \\
W_{320}=W_{20}^{(3)}, \quad W_{111}=W_{11}^{(1)}, \\
W_{211}=W_{11}^{(2)} \quad W_{311}=W_{11}^{(3)}, \\
W_{20}(\theta)=\frac{i g_{20}}{\omega \tau_{0}} q(0) e^{i \theta \omega \tau_{0}} \\
+\frac{i \overline{g_{02}}}{3 \omega \tau_{0}} \overline{q(0)} e^{-i \theta \omega \tau_{0}}+E_{1} e^{2 i \theta \omega \tau_{0}}, \\
W_{11}(\theta)=-\frac{i g_{11}}{\omega \tau_{0}} q(0) e^{i \theta \omega \tau_{0}} \\
+\frac{i \overline{g_{11}}}{\omega \tau_{0}} \overline{q(0)} e^{-i \theta \omega \tau_{0}}+E_{2},
\end{gathered}
$$

$E_{1}$

$$
\begin{aligned}
& =\left(\begin{array}{ccc}
d+2 i \omega+\frac{\beta y^{*}}{q z^{*}+1} & \frac{\beta x^{*}}{q z^{*}+1} & -\frac{q \beta x^{*} y^{*}}{\left(q z^{*}+1\right)^{2}} \\
-\frac{\beta y^{*}}{q z^{*}+1} & 2 i \omega & p y^{*}+\frac{q \beta x^{*} y^{*}}{\left(q z^{*}+1\right)^{2}} \\
0 & -c e^{-2 i \omega \tau_{0}} z^{*} & b+2 i \omega-c e^{-2 i \omega \tau_{0}} y^{*}
\end{array}\right)^{-1} \\
& \times\left(\begin{array}{l}
F_{11} \\
F_{21} \\
F_{31}
\end{array}\right),
\end{aligned}
$$

$E_{2}$

$$
=\left(\begin{array}{ccc}
d+\frac{\beta y^{*}}{q z^{*}+1} & \frac{\beta x^{*}}{q z^{*}+1} & -\frac{q \beta x^{*} y^{*}}{\left(q z^{*}+1\right)^{2}} \\
-\frac{\beta y^{*}}{q z^{*}+1} & 0 & p y^{*}+\frac{q \beta x^{*} y^{*}}{\left(q z^{*}+1\right)^{2}} \\
0 & -c z^{*} & b-c y^{*}
\end{array}\right)^{-1}\left(\begin{array}{c}
F_{12} \\
F_{22} \\
F_{32}
\end{array}\right) .
$$

Then we get

$$
\begin{gathered}
c_{1}(0)=\frac{i}{2 \omega \tau_{0}}\left(g_{20} g_{11}-2\left|g_{11}\right|^{2}-\frac{\left|g_{02}\right|}{3}\right)+\frac{g_{21}}{2}, \\
\nu_{2}=-\frac{\operatorname{Re}\left\{c_{1}(0)\right\}}{\operatorname{Re}\left\{\lambda^{\prime}\left(\tau_{0}\right)\right\}}, \\
\beta_{2}=2 \operatorname{Re}\left\{c_{1}(0)\right\}, \\
T_{2}=-\frac{\operatorname{Re}\left\{c_{1}(0)\right\}+\nu_{2} \operatorname{Re}\left\{\lambda^{\prime}\left(\tau_{0}\right)\right\}}{\omega \tau_{0}},
\end{gathered}
$$

which determine the quantities of bifurcating periodic solutions reduced on the central manifold at the critical value $\tau_{0}$. More precisely, $v_{2}$ determines the direction of Hopf bifurcation and $\beta_{2}$ determines the stability of bifurcating periodic solution. Summarizing the above discussion, we have the following main result.

Theorem 10. If $v_{2}>0\left(v_{2}<0\right)$, then the Hopf bifurcation is supercritical (subcritical) and the bifurcating periodic solutions

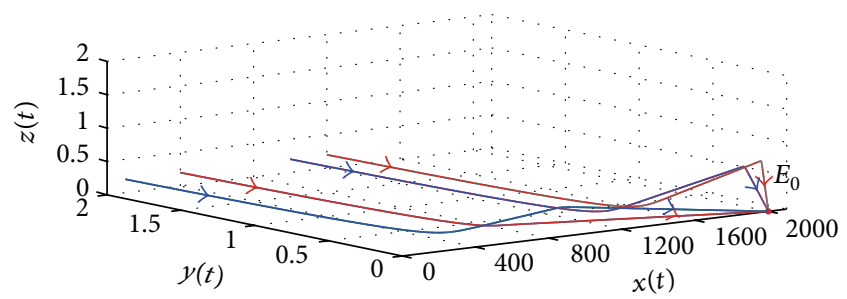

Figure 1: The phase diagram of system (2) with different initial values and different $\tau$.
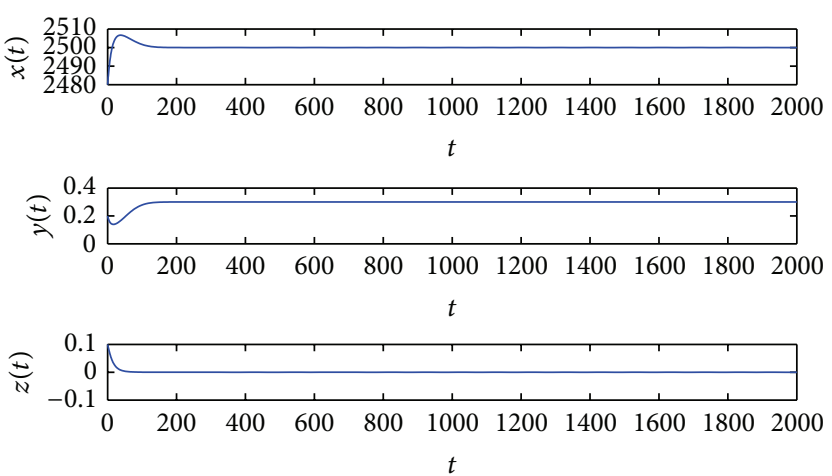

FIGURE 2: Local asymptotic stability of the immune-exhausted equilibrium $E_{1}$.

exist for $\tau>\tau_{0}\left(\tau<\tau_{0}\right)$. Moreover, $\beta_{2}$ determines the stability of the bifurcating periodic solutions. The bifurcating periodic solutions are stable (unstable) if $\beta_{2}<0\left(\beta_{2}>0\right)$.

\section{Numerical Simulation}

In order to demonstrate our results and find complex dynamic behavior of system (2), we provide numerical calculations for different birth rates of susceptible cells $s$ and time delays $\tau$. The parameter values are chosen from literatures $[14,15,20]$. If we choose the following data set: $s=200, \beta=0.002, q=0.01, a=5, b=0.1, c=0.2, d=0.1$, and $p=0.1$, then we get $R_{0}=0.8<1, E_{0}=(2000,0,0)$. Hence, by Theorem $5, E_{0}$ is globally asymptotically stable. Figure 1 illustrates this fact.

If we choose the following data set: $s=251, \beta=0.002$, $q=0.01, a=5, b=0.1, c=0.2, d=0.1$, and $p=0.1$, then we get $1<R_{0} \leqslant 1.01, E_{1}=(2500,0.3,0)$. Hence, by Theorem 4 , $E_{2}$ is local asymptotically stable. Figure 2 illustrates this fact.

In addition, we choose a set of parameters: $s=257, \beta=$ $0.002, q=0.01, a=5, b=0.1, c=0.2, d=0.1$, and $p=0.1$, then we get $R_{0}>1.01, E_{2}=(2544.7,0.5,0.5937)$, the positive real roots of (27), $x_{1}=0.00607941$ and $x_{2}=0.00194817$. It satisfies the conditions (2) of Theorem 9. We can calculate that

$$
\begin{array}{ll}
c_{1}(0)=-0.00241449-0.003121 i \\
\tau_{1}^{(0)}=8.78303, & \tau_{2}^{(0)}=37.8851, \\
\tau_{1}^{(1)}=89.3671, & \tau_{1}^{(2)}=169.951 .
\end{array}
$$



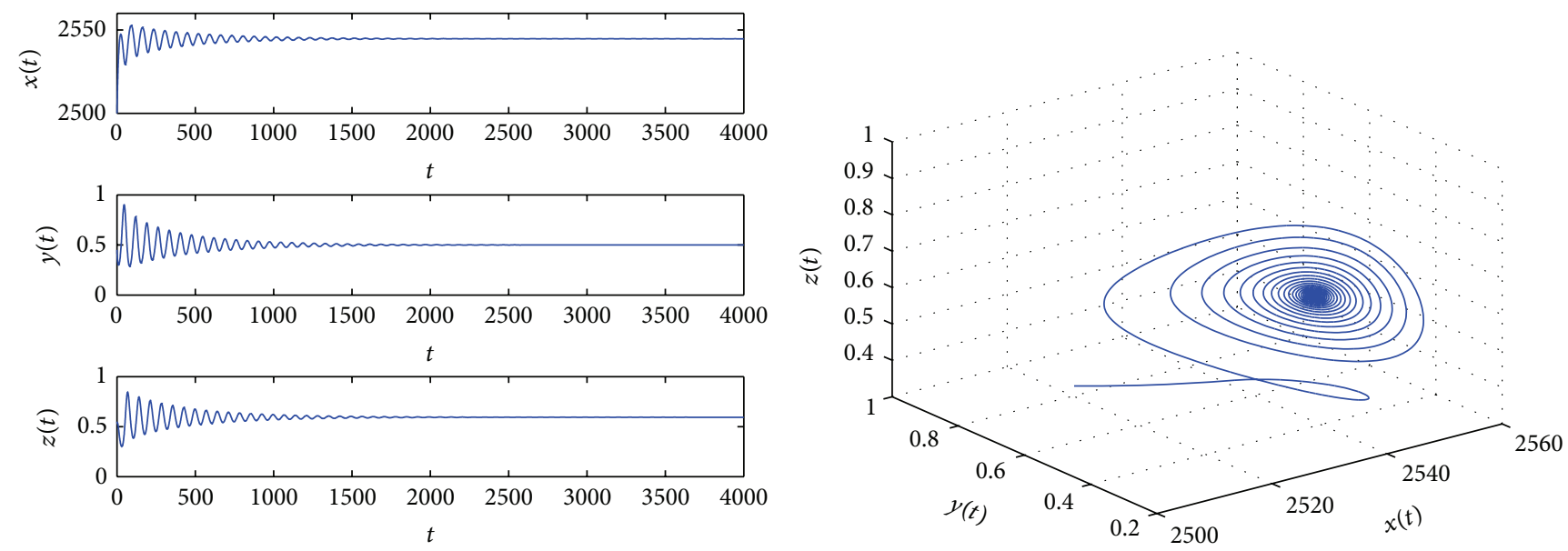

FIGURE 3: Stability of $E_{2}$ for $\tau=5$.
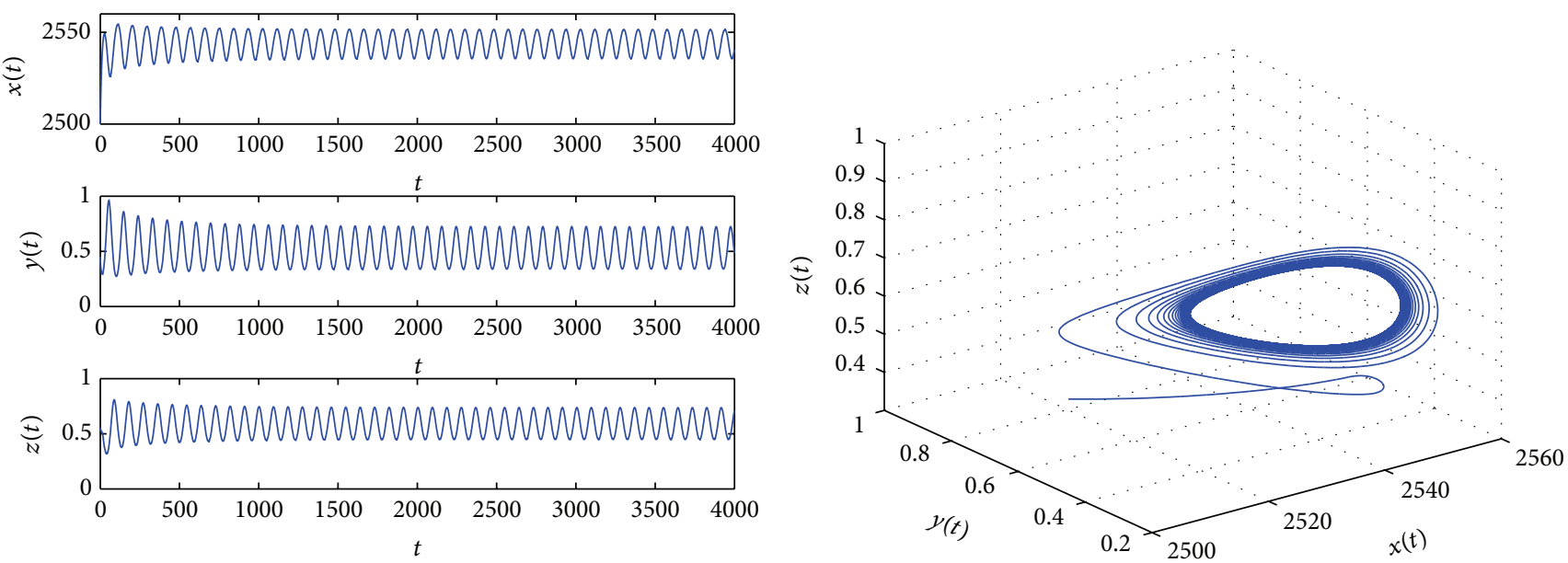

FIgURE 4: A stable bifurcating periodic solution for $\tau=13$.

By Theorem 9, we have that $E_{2}$ is stable when $\tau<$ $\tau_{1}^{(0)}$ (Figure 3). Let $\tau_{0}=\tau_{1}^{(0)}$; we can compute $c_{1}(0)=$ $-0.000199515-0.0139977 i, G^{\prime}\left(x_{1}\right)=0.000059>0$. Since $\operatorname{sign}\left[\operatorname{Re} \lambda^{\prime}\left(\tau_{0}\right)\right]=\operatorname{sign}\left[G^{\prime}\left(x_{1}\right)\right]$ and $\operatorname{Re}\left\{c_{1}(0)\right\}<0$, then $\nu_{2}>0$; according to Theorem 10 , there exist stable periodic solutions (Figure 4) for $\tau>\tau_{1}^{(0)}$ in a $\tau_{1}^{(0)}$ neighborhood. When $\tau$ exceeds $\tau_{2}^{(0)}$, we can observe that another change of stability appears (Figure 5). Since $G^{\prime}\left(x_{2}\right)=-0.00004<0$ and $\operatorname{Re}\left\{c_{1}(0)\right\}<0$ then $v_{2}>0$. Applying Theorem 10, there exist stable periodic solutions for $\tau<\tau_{2}^{(0)}$. By the same method, the stability of $E_{2}$ is changed when $\tau$ exceeds $\tau_{1}^{(1)}, \tau_{1}^{(2)}$. We can also obtain that there exist stable periodic solutions for $\tau>\tau_{1}^{(1)}$ (Figure 6). The bifurcation diagram (Figure 7) shows that the stability of $E_{2}$ is changed at $\tau_{1}^{(0)}, \tau_{2}^{(0)}$, and $\tau_{1}^{(1)}$ clearly.

As shown in Figures 3-7, for different delays in CTL response, the system is stable at a positive equilibrium when the delay is small or it is at a stable periodic oscillation when the delay is large. Our simulations demonstrated that delays may affect cell-virus and virus-immune system dynamics. These results could provide insights into many important questions such as how to further improve our mathematical model, how to better implement clinical diagnosis, and how to design and administrate more efficient medical treatment.

\section{Conclusion}

In this paper, we have studied a virus infection model with delayed nonlytic immune response. We obtain the sufficient and necessary conditions for the existence of the equilibria. Global stability of the infection-free equilibrium has been given by the Lyapunov-LaSalle type theorem. We find that the infection-free equilibrium $E_{0}$ is globally asymptotically stable if $R_{0} \leqslant 1$. In this case, the virus is finally cleared up. By analyzing corresponding characteristic equation, we obtain that the immune-exhausted equilibrium $E_{1}$ is locally asymptotically stable if $1<R_{0} \leqslant 1+b \beta / c d$. In this case, 

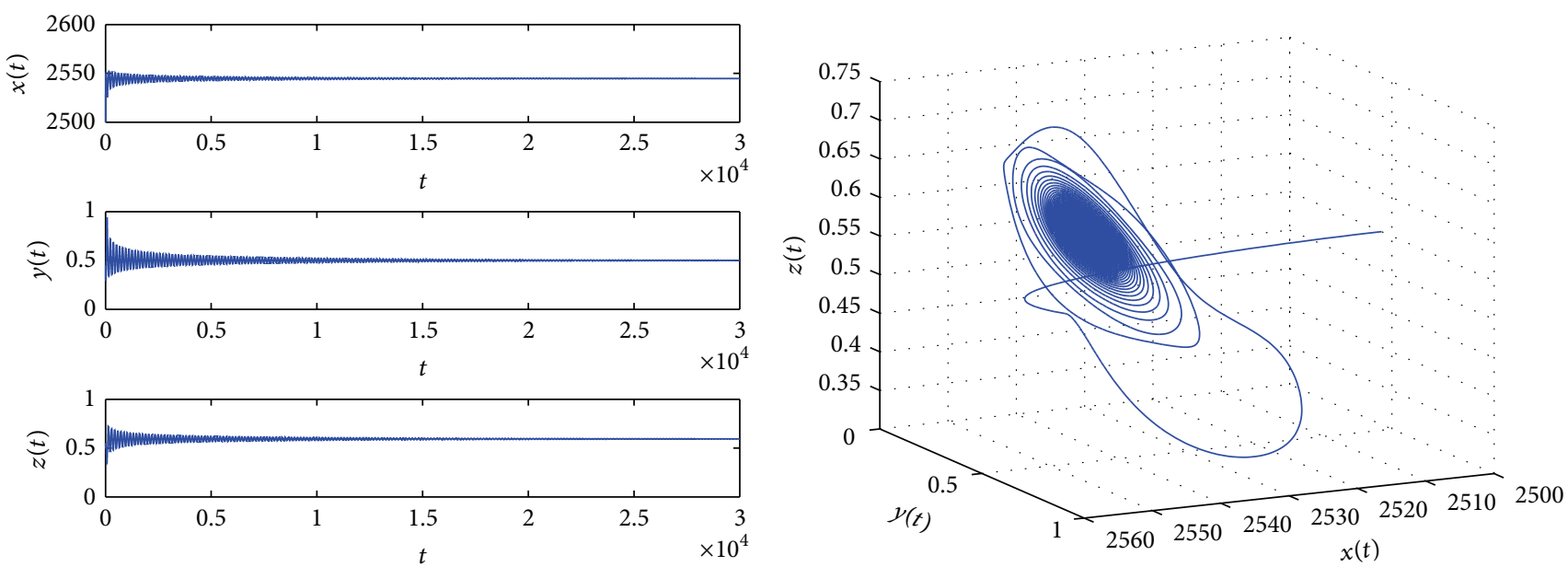

Figure 5: Stability of $E_{2}$ for $\tau=40$.
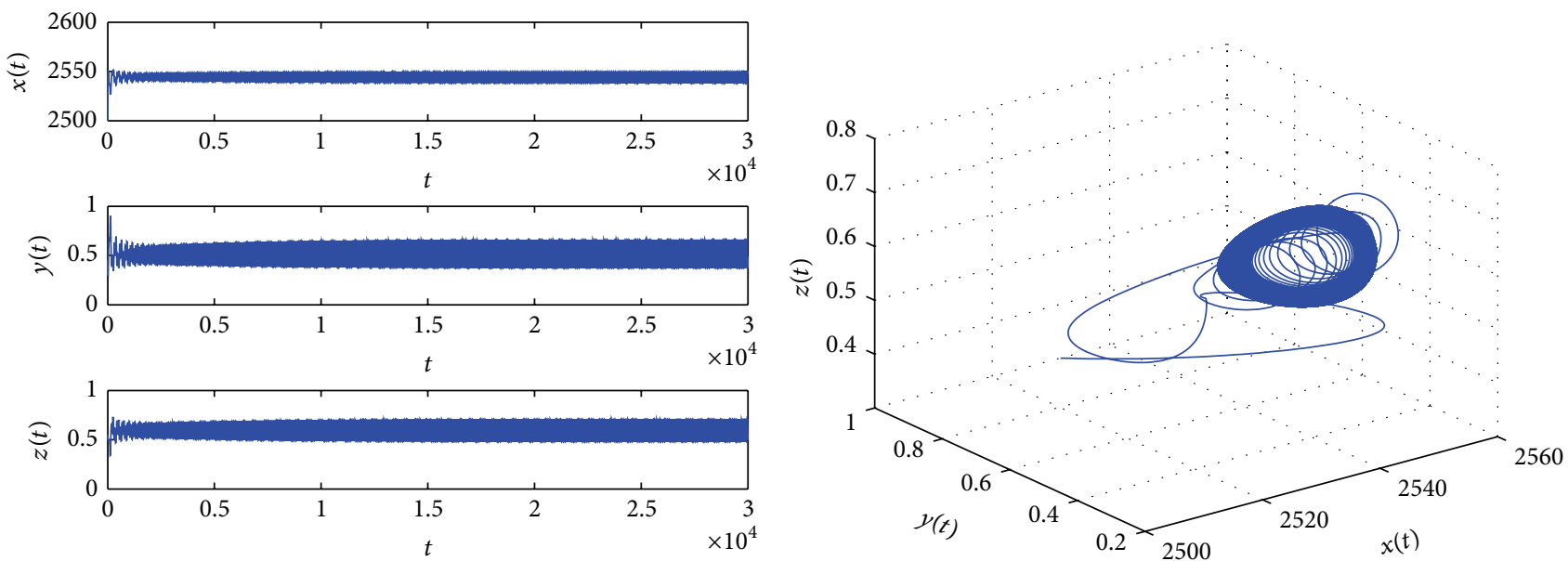

FIgURE 6: A stable bifurcating periodic solution for $\tau=95$.

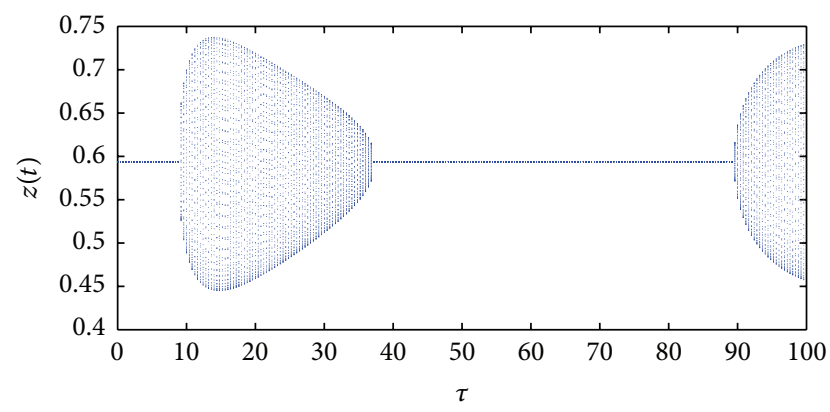

FIGURE 7: A bifurcation diagram in which $\tau$ is bifurcating parameter.

the infection becomes chronic but without CTL immune response. By choosing time delay as a bifurcation parameter, we have shown that a limit cycle emerges via Hopf bifurcation when the delay passes through the critical value $\tau_{0}$. By applying normal form theory and center manifold theorem, the direction of Hopf bifurcation and stability of bifurcating periodic solutions have been investigated. Some numerical simulations are performed to verify the correctness of theoretical analysis. Clearly, compared to the earlier studies, our analysis shows that the new time delay we introduce can change the stability of the equilibrium $E_{2}$. We would like to mention that whether the equilibrium $E_{2}$ is globally asymptotically stable when $R_{0}>1+b \beta / c d$. We leave this for our future work.

\section{Conflict of Interests}

The authors declare that there is no conflict of interests regarding the publication of this paper.

\section{References}

[1] A. S. Perelson and P. W. Nelson, "Mathematical analysis of HIV1 dynamics in vivo," SIAM Review, vol. 41, no. 1, pp. 3-44, 1999.

[2] P. De Leenheer and H. L. Smith, "Virus dynamics: a global analysis," SIAM Journal on Applied Mathematics, vol. 63, no. 4, pp. 1313-1327, 2003. 
[3] M. A. Nowak and R. M. May, Virus Dynamics: Mathematical Principles of Immunol ogy and Virology, Oxford University Press, Oxford, UK, 2000.

[4] R. V. Culshaw and S. Ruan, "A delay-differential equation model of HIV infection of $\mathrm{CD}^{4+}$ T-cells," Mathematical Biosciences, vol. 165, no. 1, pp. 27-39, 2000.

[5] C. J. Browne and S. S. Pilyugin, "Periodic multidrug therapy in a within-host virus model," Bulletin of Mathematical Biology, vol. 74, no. 3, pp. 562-589, 2012.

[6] L. Rong, Z. Feng, and A. S. Perelson, "Emergence of HIV1 drug resistance during antiretroviral treatment," Bulletin of Mathematical Biology, vol. 69, no. 6, pp. 2027-2060, 2007.

[7] A. S. Perelson, D. E. Kirschner, and R. de Boer, "Dynamics of HIV infection of $\mathrm{CD}^{+}{ }^{+} \mathrm{T}$ cells," Mathematical Biosciences, vol. 114, no. 1, pp. 81-125, 1993.

[8] R. J. de Boer and A. S. Perelson, "Towards a general function describing T cell proliferation," Journal of Theoretical Biology, vol. 175, no. 4, pp. 567-576, 1995.

[9] R. J. De Boer and A. S. Perelson, "Target cell limited and immune control models of HIV infection: a comparison," Journal of Theoretical Biology, vol. 190, no. 3, pp. 201-214, 1998.

[10] Y. Ji, L. Min, Y. Zheng, and Y. Su, "A viral infection model with periodic immune response and nonlinear CTL response," Mathematics and Computers in Simulation, vol. 80, no. 12, pp. 2309-2316, 2010.

[11] M. A. Nowak and C. R. M. Bangham, "Population dynamics of immune responses to persistent viruses," Science, vol. 272, no. 5258, pp. 74-79, 1996.

[12] X. Song, S. Wang, and J. Dong, "Stability properties and Hopf bifurcation of a delayed viral infection model with lytic immune response," Journal of Mathematical Analysis and Applications, vol. 373, no. 2, pp. 345-355, 2011.

[13] K. Wang, W. Wang, and X. Liu, "Viral infection model with periodic lytic immune response," Chaos, Solitons and Fractals, vol. 28, no. 1, pp. 90-99, 2006.

[14] C. Bartholdy, J. P. Christensen, D. Wodarz, and A. R. Thomsen, "Persistent virus infection despite chronic cytotoxic Tlymphocyte activation in gamma interferon-deficient mice infected with lymphocytic choriomeningitis virus," Journal of Virology, vol. 74, no. 22, pp. 10304-10311, 2000.

[15] D. Wodarz, J. P. Christensen, and A. R. Thomsen, "The importance of lytic and nonlytic immune responses in viral infections," Trends in Immunology, vol. 23, no. 4, pp. 194-200, 2002.

[16] N. Burić, M. Mudrinic, and N. Vasović, "Time delay in a basic model of the immune response," Chaos, Solitons \& Fractals, vol. 12, no. 3, pp. 483-489, 2001.

[17] A. A. Canabarro, I. M. Gléria, and M. L. Lyra, "Periodic solutions and chaos in a non-linear model for the delayed cellular immune response," Physica A: Statistical Mechanics and Its Applications, vol. 342, no. 1-2, pp. 234-241, 2004.

[18] K. Wang, W. Wang, H. Pang, and X. Liu, "Complex dynamic behavior in a viral model with delayed immune response," Physica D, vol. 226, no. 2, pp. 197-208, 2007.

[19] M. Y. Li and H. Shu, "Global dynamics of a mathematical model for HTLV-I infection of CD $4^{+} \mathrm{T}$ cells with delayed CTL response," Nonlinear Analysis: Real World Applications, vol. 13, no. 3, pp. 1080-1092, 2012.

[20] Q. Xie, D. Huang, S. Zhang, and J. Cao, "Analysis of a viral infection model with delayed immune response," Applied Mathematical Modelling, vol. 34, no. 9, pp. 2388-2395, 2010.
[21] T. Wang, Z. Hu, and F. Liao, "Stability and Hopf bifurcation for a virus infection model with delayed humoral immunity response," Journal of Mathematical Analysis and Applications, vol. 411, no. 1, pp. 63-74, 2014.

[22] B. D. Hassard, N. D. Kazarinoff, and Y. H. Wan, Theory and Applications of Hopf Bifurcation, Cambridge University Press, Cambridge, UK, 1981. 


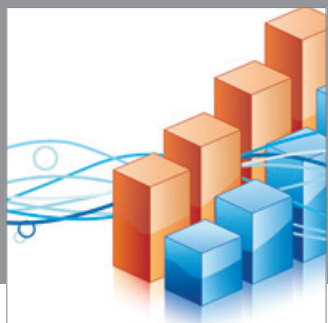

Advances in

Operations Research

mansans

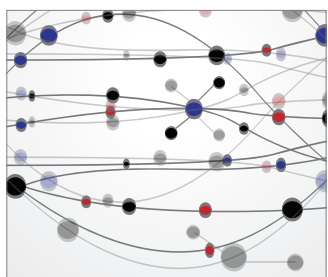

The Scientific World Journal
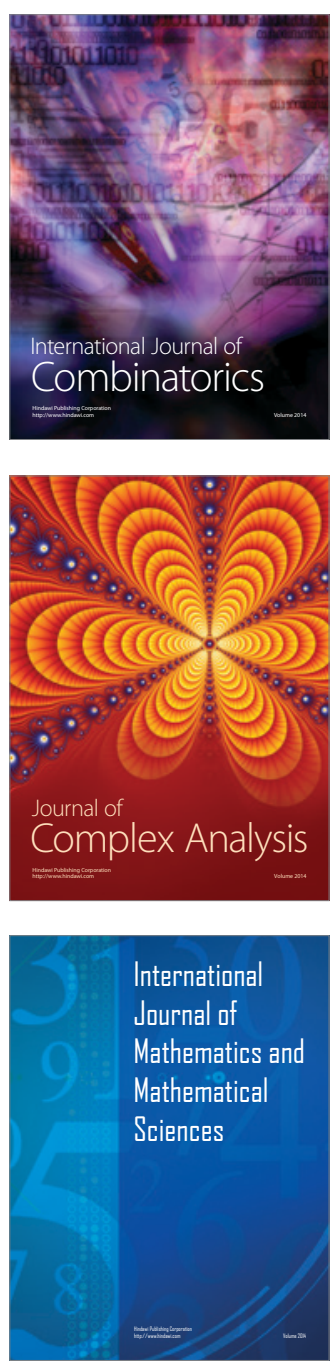
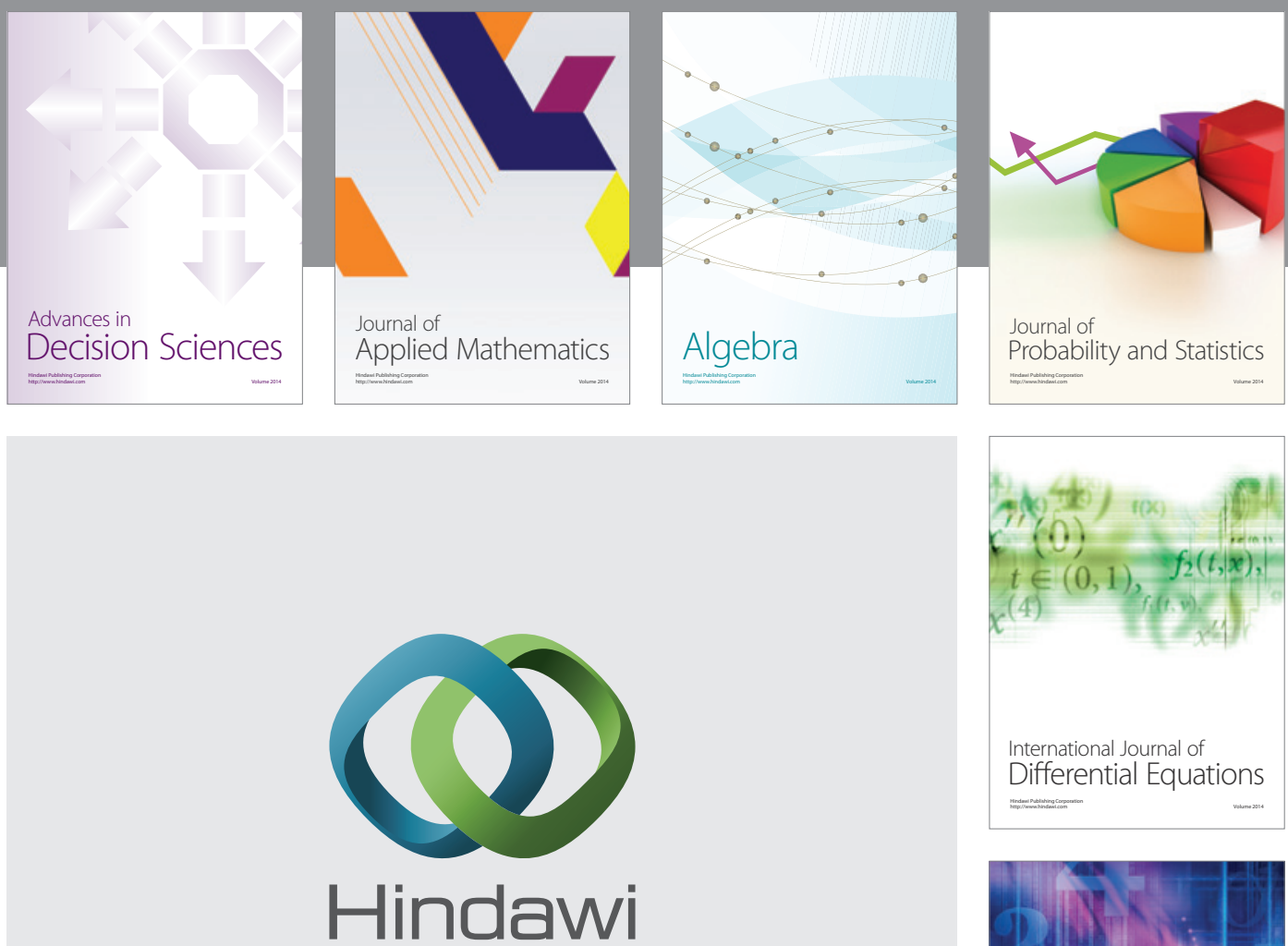

Submit your manuscripts at http://www.hindawi.com
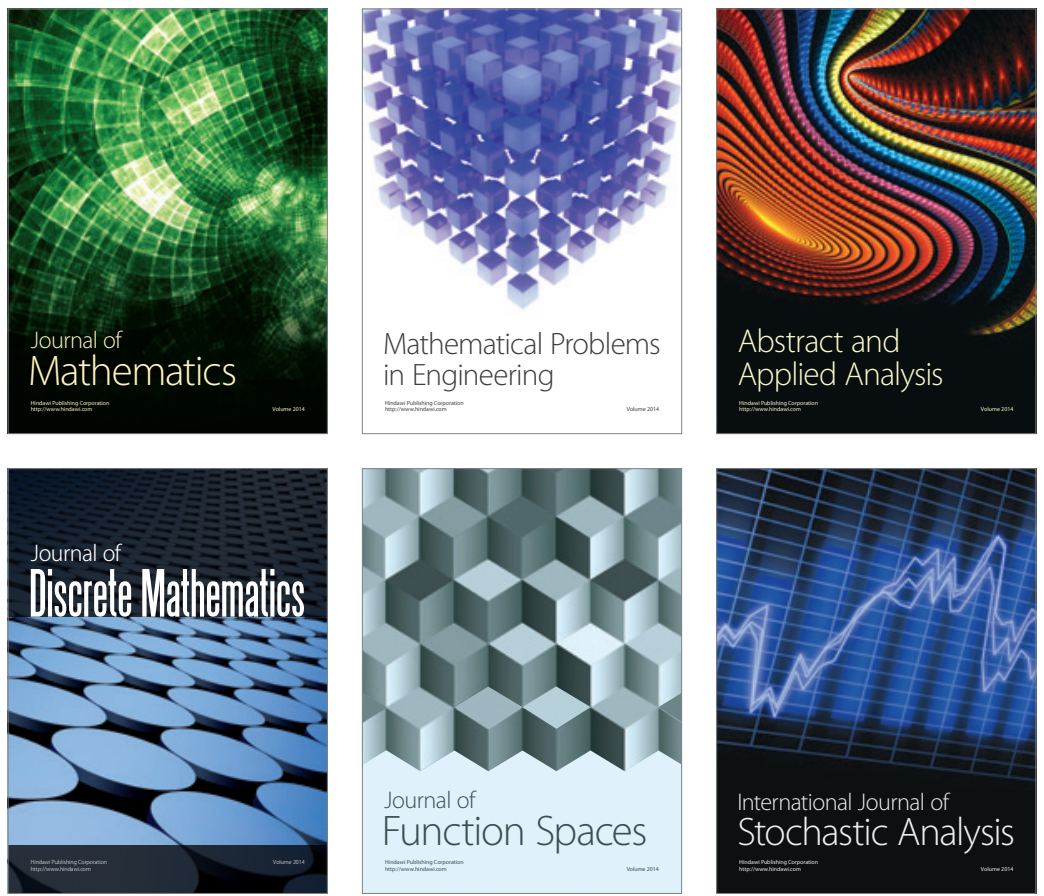

Journal of

Function Spaces

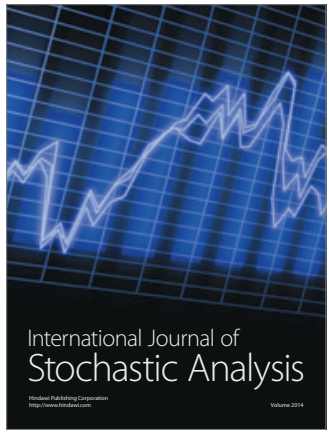

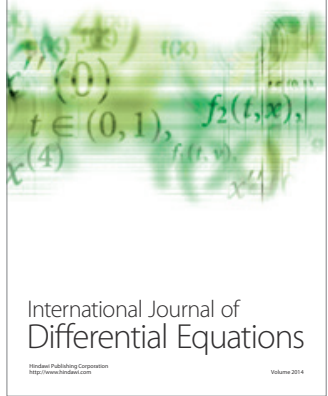
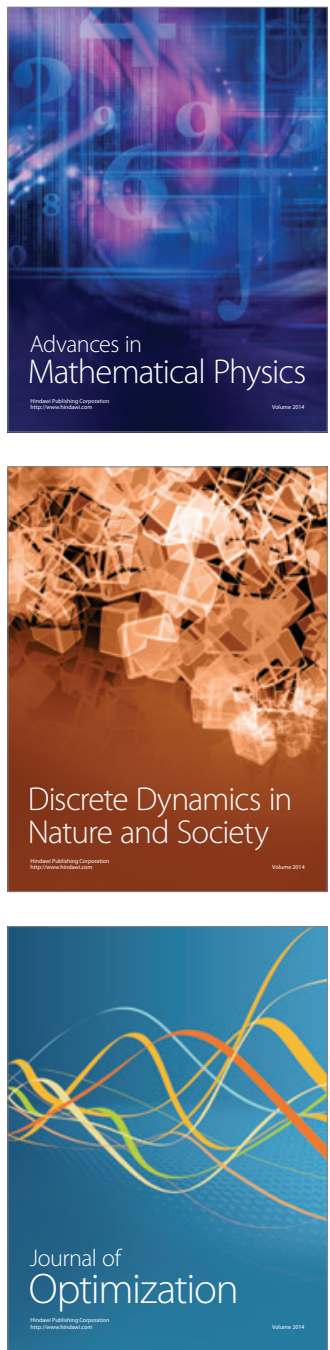